\title{
Papierelektrophoretische Untersuchungen von Rattenseren bei experimenteller diätetischer Lebernekrose
}

\author{
Von \\ G. BRENNER und H. KREMER \\ Aus der Universitäts-Frauenklinik Bonn (Direktor: Prof. Dr. H. Siebke)
}

(Der Schriftleitung zugegangen am 8. Juli 1963)

\begin{abstract}
Mittels Papierelektrophorese wurden die Bluteiweißfraktionen in Rattenseren bestimmt. Die in den Seren von Ratten mit alimentärer Lebernekrose gefundenen Veränderungen wurden mit den Werten gesunder Ratten gleicher Zucht statistisch verglichen. Sowohl der Abfall des Albumins um rund 30\% der Norm als auch das Ansteigen der $\alpha_{1^{-}}, \alpha_{2^{-}}$, $\beta_{1}$ - und $\beta_{2}$-Globuline sind gegenüber derNorm signifikant. Es wurde kurz auf die Ähnlichkeit mit den Veränderungen im Serumeiweißbild bei der menschlichen Eklampsie hingewiesen.

Blood protein fractions in rat sera were measured by paper electrophoresis. Changes found in the sera of rats with alimentary liver necrosis were compared statistically with values from healthy rats of the same litter. A fall in albumin to about $30 \%$ of the norm and an increase of $\alpha_{1-}, \alpha_{2}-, \beta_{1}$ - and $\beta_{2}$-globulins compared with the norm are significant. The similarity to blood protein changes in human eclampsia is briefly discussed.
\end{abstract}

Zur Klärung der Ätiologie der experimentellen diätetischen Lebernekrose der Ratte sind in den letzten Jahren zahlreiche Untersuchungen durchgeführt worden (1). Hierbei standen besonders die biochemischen Veränderungen in der geschädigten Leber selbst und auch die Auswirkungen auf den Gesamtorganismus im Vordergrund des Interesses. Die mit sogenannten "nekrogenen“ Diäten experimentell darstellbare Lebernekrose der Ratte ist während einer langen Diätperiode nur stoffwechselfunktionell nachweisbar. Erst kurz vor dem Tode kommt es in der Leber zu den massiven degenerativen Veränderungen, die zum plötzlichen Zusammenbruch führen. - Uns schien es von großem Interesse, die Auswirkungen dieser teilweise sehr tiefgreifenden Schädigung des Leberzell- und Leberzellkerngefüges auf die Serumeiweißfraktionen derart geschädigter Ratten zu untersuchen.

In früheren Veröffentlichungen wurden bei Ratten, die eine fettreiche und Vitamin-E-freie Diät erhielten, keine typischen Veränderungen des Serumeiweißbildes gefunden (2). In Vorversuchen konnten wir feststellen, $\mathrm{da} \beta$ vor der Bildung massiver nekrotischer Leberschäden keine signifikanten Abweichungen der Serumeiweißfraktionen von den Normalwerten auftreten.

\section{Methodik}

\section{a) Versucbstiere und Versucbsdiät}

Die Untersuchungen wurden mit jungen, infantilen Albinoratten im Gewicht von $50-80 \mathrm{~g}$ durchgeführt. Sie entstammten unserer klinikseigenen Zucht und waren durch mehrere Generationen streng ingezüchtet, so da $\beta$ erbmäßige Abweichungen innerhalb des Tiermaterials auf ein Minimum beschränkt waren. Die Aufteilung der einzelnen Würfe erfolgte in der Art, daß die Zusammensetzung der zu vergleichenden Gruppen in Geschlecht, Anzahl der Geschwistertiere und dem Durchschnittsgewicht übereinstimmte. Während des Versuches wurden die Ratten in Einzelkäfigen gehalten. Fütterung und Tränkung erfolgten täglich ad libitum. Die Blutentnahme wurde in Âthernarkose durch Herzpunktion vor- genommen. Nach der Blutentnahme wurden die Tiere getötet und auf pathologische Veränderungen an Leber, Nieren und Milz hin untersucht.

Als lebernekrose-auslösende Diät wählten wir eine Hefediät (Torula utilis) vom Nährstoffverhältnis 1:10 (etwa $10 \%$ verdauliches Hefeprotein), die von Hock und Fink (3) erstmals zur Erzeugung von Lebernekrosen bei der Ratte verwendet wurde. Die Zusammensetzung ist aus Tabelle 1 zu ersehen:

Tab. 1

Zusammensetzung der Diät (Werte in \% Trockensubstanz)

\begin{tabular}{lr}
\hline Hefe: & 16,84 \\
Bierhefe: & 1,45 \\
Lebertran: & 2,27 \\
Salzgemisch & ): \\
Weizenstärke: & 3,55 \\
prim. Ca-phosphat: & 74,05 \\
& 1,84
\end{tabular}

1) Nach McColitum und Simmonds $(4,5)$.

Bis auf das ennährungsphysiologisch unterwertige Hefeeiweiß war die Diät für die Ratte suffizient. Die Diät wurde in Form von Keksen verfüttert. Die als Vergleichsreihe mitgeführten „Normaltiere“ erhielten „Latz"-Fertigfutter.

\section{b) Elektrophorese}

Die Fraktionierung der Rattenseren erfolgte mittels Papierelektrophorese nach GrassmanN und HaNNIG (6). Unsere Anordnung bestand aus einem Netzanschlußgerät und mehreren Elphor-HElektrophoresekammern mit Labyrinthsystem. Als Puffersystem wählten wir nach etlichen Vorversuchen einen Veronalnatrium/ Natriumacetatpuffer (MrCHAELIS) mit einem $\mathrm{pH}$ von 8,6 und einer Ionenstärke von 0,10 . Die Laufzeit betrug $15 \mathrm{Stdn}$. bei einer stabilen Spannung von 110 Volt. Die Elektrophoresen wurden auf Schleicher- und Schüll-Papier 2043 a Mgl. in einem thermostabilen Raum bei einer Temperatur von $20^{\circ}$ durchgeführt. Die Papierstreifen wurden nach der Elektrophorese mit Amidoschwarz $10 \mathrm{~B}$ angefärbt und nach Entfärbung mit einer Lösung aus Methanol und $10 \%$ Eisessig mit einem Gemisch aus einem Teil $\alpha$-Bromnaphthalin und einem Teil Paraffinöl im Vakuum transparent gemacht. Die Auswertung der zwischen 2 Glasplatten eingedeckten Streifen erfolgte in dem vollautomatischen Ausmeßgerät (Extinktionsschreiber mit gekoppeltem Intregralschreiber) der Fa. C. Zeiss. 


\section{c) Statistische Auswertung}

Bei einem Vergleich zweier oder mehreter parallellaufender Versuchsreihen besteht ohne weiteres keine Möglichkeit zu beurteilen, ob eine auftretende Abweichung noch in den Bereich der Zufallsschwankungen (Streuung) fällt, oder ob der Befund auf einen signifikanten Unterschied der zu vergleichenden Gruppen schließen läßt. Aus diesem Grunde wurden bei unseren Versuchsauswertungen statistische Prüfungsmethoden herangezogen. Die von Koller (7) vorgeschlagene Methode, die hier angewandt wurde, geht von der Berechnung der ,mittleren Abweichung“" aus:

$$
\sigma=\sqrt{\frac{\Sigma \mathrm{d}^{2}}{\mathrm{n}-1}}
$$

Um nun festzustellen, ob die zu vergleichenden Reihen als Stichproben aus derselben Grundgesamtheit aufgefaßt werden können, wird der ,mittlere Fehler der Differenz der Mittelwerte" nach der Formel:

$$
\sigma_{\text {Diff. }}=\sqrt{\frac{\sigma_{1}^{2}}{\mathrm{n}_{2}}+\frac{\sigma_{2}^{2}}{\mathrm{n}_{1}}}
$$

berechnet. Darin bedeuten:

$\mathrm{d} \quad=$ Differenz der Einzelwerte vom Mittelwert

n = Anzahl der Beobachtungswerte, d. h. der Versuchstiere

$\sigma=$ mittlere Abweichung vom Mittelwert

$\sigma_{\text {Diff, }}=$ mittlerer Fehler der Differenz der Mittelwerte.

Ist nun die Differenz der durchschnittlichen Beobachtungswerte der beiden Versuchsreihen $\left(M_{1}-M_{2}\right)$ größer als $\sigma_{\text {Diff, multipliziert }}$ mit dem Faktor $t$ (STudent scher $t$-Test), dessen Tafelwert durch die Anzahl der Freiheitsgrade $\left(m=n_{1}+n_{2}-2\right)$ gegeben ist, so ist der Unterschied in den beiden Versuchsgruppen als signifikant anzisehen.

\section{Ergebnisse}

a) Normaltiere

$\mathrm{Da}$ das Ergebnis unserer papierelektrophoretischen Untersuchungen einerseits wesentlich abhängig ist von der angewandten Technik (8), andererseits auch von Rasse, Zucht (9) und Alter (10) der Versuchstiere, mußten zunächst Normalwerte an unbehandelten Ratten derselben Zucht bzw. an Geschwistertieren ermittelt werden. Erst dann konnten unter strenger Beibehaltung aller Einzelvorgänge der Versuchsanordnung Veränderungen im Serumeiweißbild beurteilt werden. Diese Tatsachen erklären auch die teilweise sehr beträchtlichen Unterschiede, die bei früheren Untersuchungen verschiedener Autoren, insbesondere bei den Albumin- und $\gamma$-Globulingehalten von Rattenseren auftraten. In der folgenden Tabelle 2 sind einige Normalwerte für gesunde Ratten zusammengestellt. Unsere Normalwerte, die wir an 30 Ratten (15 o und $\left.15 \sigma^{\circ}\right)$ bestimmten, zeigen eine gute Übereinstimmung unterein-

Tab. 2

\begin{tabular}{|c|c|c|c|c|c|}
\hline Autor & Albumin & $\alpha_{1^{-}}$ & $\alpha_{2^{-}}$ & $\beta$ & $\begin{array}{c}\gamma \text {-Globu- } \\
\text { line }\end{array}$ \\
\hline CAGLI (11) & 52,2 & 15,2 & 5,4 & 18,2 & 8,6 \\
\hline Martini (12) & 42,0 & 10,7 & 10,8 & 18,6 & 17,8 \\
\hline \multirow[t]{2}{*}{ BERG (13) } & 46,5 & 12,5 & 8,8 & 21,1 & 11,5 \\
\hline & {$[\sigma= \pm 5,2$} & $\pm 2,5$ & $\pm 1,8$ & $\pm 2,6$ & $\pm 5,9]$ \\
\hline \multirow{2}{*}{ Piliero (14) } & 58,9 & 8,9 & 6,2 & 16,7 & 9,3 \\
\hline & {$[\sigma= \pm 3,8$} & $\pm 1,5$ & $\pm 0,6$ & $\pm 1,7$ & $\pm 1,2]$ \\
\hline Gleason (15) & $\overline{88,6}$ & 1,2 & 0,4 & 6,9 & 2,9 \\
\hline SchMIDt (16) & 55,0 & 11,6 & 2,8 & 11,1 & 18,9 \\
\hline
\end{tabular}

Normalwerte gesunder Ratten in rel. Prozenten.
Tab. 3

\begin{tabular}{|c|c|c|c|c|c|c|}
\hline & Albumin & $\alpha_{1}-$ & $\alpha_{2^{-}}$ & $\beta_{1^{-}}$ & $\beta_{2^{-}}$ & $\gamma$-Globuline \\
\hline 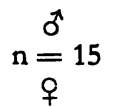 & 49,1 & 11,7 & 8,0 & 3,5 & 16,8 & 10,7 \\
\hline $\mathrm{n}=15$ & 51,8 & 12,1 & 7,4 & 2,9 & 15,9 & 10,9 \\
\hline $\begin{array}{l}\text { Gesamt } \\
\mathrm{n}=30\end{array}$ & $\begin{array}{r}50,5 \\
{[\sigma= \pm 4,0}\end{array}$ & $\begin{array}{r}11,9 \\
\pm 1,6\end{array}$ & $\begin{array}{r}7,7 \\
\pm 1,6\end{array}$ & $\begin{array}{r}3,2 \\
\pm 0,6\end{array}$ & $\begin{array}{r}16,4 \\
\pm 2,4\end{array}$ & $\begin{array}{c}10,8 \\
\pm 2,8]\end{array}$ \\
\hline
\end{tabular}

Normalwerte gesunder Ratten in rel. Prozenten.

ander. Wie aus der Tabelle $3 \mathrm{zu}$ ersehen ist, liegt die mittlere Abweichung vom Mittelwert $(\sigma)$ in der von den meisten Autoren angegebenen Größenordnung.

Bei der Auswertung der Elektrophoresestreifen konnten wir vor der eigentlichen $\beta$-Fraktion, die im Diagramm ein für die Ratte charakteristisches Bild zeigt, oft schon makroskopisch erkennbar eine weitere Fraktion ausmachen, die wir als $\beta_{1}$-Globulin bezeichneten.

\section{b) Tiere mit Lebernekrose}

Eine große Schwierigkeit bei unserer Untersuchung war die Festlegung des Zeitpunktes der Blutentnahme bei den mit Hefe-Diät ernährten Ratten. Wie eingangs erwähnt, treten die degenerativen Leberveränderungen erst kurz vor dem Tode auf. Bei vor diesem Zeitpunkt getöteten Ratten zeigten sich, wie wir wiederholt feststellen konnten, keine oder nur unerhebliche Veränderungen im Serumeiweißbild. Andererseits konnte bei bereits toten Tieren kein einwandfreies Serum mehr gewonnen werden. Wir entschlossen uns daher, die Tiere laufend $\mathrm{zu}$ beobachten und die Blutentnahme erst kurz vor dem Exitus in Äthernarkose vorzunehmen. Das prämortale Stadium ist gekennzeichnet durch einen allgemeinen Kümmerzustand und Verweigerung der Futteraufnahme; die Tiere zeigen ein dünnes, nicht mehr anliegendes Haarkleid, eine verminderte Reaktion auf äußere Reize sowie später Seitenlage und letztlich eine tiefe Bewußtlosigkeit.

Die Bestimmung der Proteinfraktionen im Serum von Ratten mit Lebernekrose ergab folgende Werte:

Tab. 4

Serumproteinfraktionen bei Ratten mit Lebernekrose in rel. Prozenten.

\begin{tabular}{lrrrrrr}
\hline & Albumin & $\alpha_{1^{-}}$ & $\alpha_{2^{-}}$ & $\beta_{1^{-}}$ & $\beta_{2^{-}}$ & $\begin{array}{c}\gamma \text {-Globu- } \\
\text { line }\end{array}$ \\
\hline $\begin{array}{l}\sigma \\
\mathrm{n}=15\end{array}$ & 35,8 & 16,2 & 9,7 & 4,7 & 20,1 & 13,3 \\
$\stackrel{\mathrm{n}}{=15}$ & 36,1 & 16,5 & 11,6 & 4,9 & 17,9 & 12,5 \\
\hline $\begin{array}{l}\text { Gesamt } \\
\mathrm{n}=30\end{array}$ & {$[\sigma= \pm 4,2 \pm 2,3$} & $\pm 3,2 \pm 1,7$ & $\pm 3,5$ & $\pm 3,8]$ \\
\hline
\end{tabular}

Tab. 5

Veränderungen der Fraktionswerte bei den Nekroseratten in

\begin{tabular}{|c|c|c|c|c|c|}
\hline Albumin & $\alpha_{1-}$ & $\alpha_{2}-$ & $\beta_{1^{-}}$ & $\beta_{2^{-}}$ & $\gamma$-Globuline \\
\hline $\begin{array}{l}0 \stackrel{2}{-} 27 \\
\text { 市 }\end{array}$ & $\begin{array}{l}+39 \\
+36\end{array}$ & $\begin{array}{l}+21 \\
+57\end{array}$ & $\begin{array}{l}+34 \\
+69\end{array}$ & $\begin{array}{l}+20 \\
+13\end{array}$ & $\begin{array}{l}+24 \\
+15\end{array}$ \\
\hline
\end{tabular}
Prozent gegenüber den Normalwerten 
Aus den Tabellen 4 und 5 ergibt sich zusammenfassend: Die Albuminfraktion sinkt bei den Nekroseratten um etwa $30 \%$ der Norm ab, während alle Globuline ansteigen.

Tab. 6

Statistische Auswertung. ( $I=$ Normaltiere; $I I=$ Tiere mit Lebernekrose).

\begin{tabular}{|c|c|c|c|c|c|c|c|c|c|}
\hline \multicolumn{2}{|c|}{ Fraktion } & & \multirow{2}{*}{$\begin{array}{l}n \\
30 \\
29\end{array}$} & \multirow{2}{*}{$\begin{array}{c}\text { M } \\
50,5 \\
35,9\end{array}$} & \multirow{2}{*}{$\frac{\sigma_{\text {Difr. }}}{1,06}$} & \multirow{2}{*}{$\begin{array}{c}\mathrm{t} \\
3,14\end{array}$} & \multirow{2}{*}{$\frac{\begin{array}{c}\sigma_{\text {DIff. }} \\
\mathrm{x} \mathrm{t}^{-}\end{array}}{3,32}$} & \multirow{2}{*}{$\begin{array}{r}M_{1}- \\
M_{2} \\
14,5\end{array}$} & \multirow{2}{*}{$\begin{array}{c}\begin{array}{r}\text { Sig- } \\
\text { nifi- } \\
\text { kanz }\end{array} \\
+\end{array}$} \\
\hline Albumin & & & & & & & & & \\
\hline Globuline & I & $\begin{array}{l}1,59 \\
2,32\end{array}$ & $\begin{array}{l}30 \\
30\end{array}$ & $\begin{array}{l}11,9 \\
16,4\end{array}$ & 0,51 & 3,13 & 1,60 & 4,5 & + \\
\hline$\alpha_{2}$ & & $\begin{array}{l}1,61 \\
3,23\end{array}$ & $\begin{array}{l}30 \\
30\end{array}$ & $\begin{array}{r}7,7 \\
10,6\end{array}$ & 0,66 & 3,13 & 2,07 & 2,9 & + \\
\hline$\beta_{1}$ & $\begin{array}{l}\text { I } \\
\text { II }\end{array}$ & $\begin{array}{l}0,57 \\
1,68\end{array}$ & $\begin{array}{l}30 \\
30\end{array}$ & $\begin{array}{l}3,2 \\
4,8\end{array}$ & 0,32 & 3,13 & 1,00 & 1,6 & + \\
\hline$\beta_{2}$ & $\begin{array}{l}\text { I } \\
\text { II }\end{array}$ & $\begin{array}{l}2,39 \\
3,48\end{array}$ & $\begin{array}{l}30 \\
30\end{array}$ & $\begin{array}{l}16,4 \\
19,0\end{array}$ & 0,77 & 3,13 & 2,41 & 2,6 & + \\
\hline$\gamma$ & $\begin{array}{l}\text { I } \\
\text { II }\end{array}$ & $\begin{array}{l}2,79 \\
3,82\end{array}$ & $\begin{array}{l}30 \\
30\end{array}$ & $\begin{array}{l}10,8 \\
12,9\end{array}$ & 0,86 & 3,13 & 2,70 . & 2,1 & - \\
\hline
\end{tabular}

Die statistische Auswertung (Tab. 6) zeigt, daß alle Fraktionen mit Ausnahme des $\gamma$-Globulins eine signifikante Veränderung gegenüber den Normalwerten aufweisen. Die größere mittlere Abweichung vom Mittelwert $(\sigma)$ bei den Nekrosetieren ist nicht methodisch bedingt, sondern liegt daran, daß die einzelnen Tiere Leberschäden unterschiedlichen Ausmaßes aufwiesen. Wir konnten immer wieder feststellen, daß bei ausgedehnten Nekrosen auch jeweils die größten Veränderungen im Bluteiweißbild auftraten. Alle Tiere, die in Tabelle 4 erfaßt wurden, zeigten eindeutige pathologische Leberveränderungen, die schon makroskopisch als typische alimentäre Lebernekrose zu identifizieren waren.
Zwischen den männlichen und weiblichen Tieren treten mit Ausnahme der erhöhten $\alpha_{2}$ - und $\beta_{1}$-Fraktionen bei den weiblichen Tieren keine nennenswerten Unterschiede auf. Interessant in diesem Zusammenhang ist die Tatsache, da $\beta$ bei getrennter statistischer Auswertung diese erhöhten $\alpha_{2}$ - und $\beta_{1}$-Fraktionen bei den weiblichen Tieren eine Signifikanz aufweisen, während bei den männlichen Tieren für diese Fraktionen keine Signifikanz zu ermitteln ist. Beide Geschlechter zusammen ergeben dann bedingt durch die höhere Tierzahl einen gesicherten Unterschied zu den Normalwerten.

Die bei den Nekroseratten auftretende Verschiebung im Bluteiweißbild ähnelt sehr stark den Veränderungen, die von verschiedenen Autoren bei der menschlichen Eklampsie gefunden wurden (17, 18, 19). In der Arbeit von GöRETZLEHNER (20) heißt es: „Unsere Untersuchungen zeigen bei allen Toxikosegruppen eine Verminderung der Serumproteine und Albumine, die bei der Präeklampsie und Eklampsie am stärksten ist. Parallel dazu kommt es zu einer weiteren Erhöhung der $\alpha_{1}^{-}, \alpha_{2^{-}}$und $\beta$-Globuline... Keinerlei pathologische Veränderungen zeigen die $\gamma$-Globuline. “ Diese Ähnlichkeit der Veränderungen im Bluteiweißbild ist keineswegs überraschend, wenn man an die Veröffentlichungen von Dobberstein und Hock (21) denkt. Die Autoren wiesen schon im Jahre 1944 auf die große Ähnlichkeit der alimentären Lebernekrose der Ratte mit dem besonders Leber und Nieren betreffenden Sektionsbild bei der Eklampsie der schwangeren Frau hin.

Eine unserer nächsten Aufgaben wird es sein, die von uns in einer früheren Untersuchung (22) gefundene Einwirkung des Östradiols-17 $\beta$ (Schutzwirkung) bzw. des Östriols (verstärkende Wirkung) auf die alimentäre Lebernekrose der Ratte durch elektrophoretische Bestimmungen der Bluteiweißfraktionen zu studieren.

\section{Literatur}

1. Strunz, K. und A. Hock. Die experimentelle diätetische Lebernekrose, Steinkopff-Verlag, Darmstadt (1960). - 2. Bock, A., Vet. Diss., Gießen (1955). - 3. Hock A. und H. Fink, HoppeSeyler's Z. Physiol. Chemie, 279, 187 (1943). - 4. Collum v.Mc., E., c. s., J. biol. Chemistry 33, 55 (1918). - 5. Collum v. Mc., E. und N. Snmmonds, The Newer Knowledge of Nutrition, 3. Auflage, Berlin und Wien (1928). - 6. GrassmanN, W. und K. Hannig, Hoppe-Seyler's Z. physiol. Chem. 290, 1 (1952). 7. Koller, S., Graphische Tafeln zur Beurteilung statistischer Zahlen, 3. Auflage, Steinkopff-Verlag, Darmstadt (1953). - - 8. WuNDERLY, CH., Die Papierelektrophorese, Sauerländer \& Co., Frankfurt-Main (1959). - 9. WinkLER, C. und G. Paschke, Z. Naturforsch. 10b, 478 (1955). - 10. U UEL, H., Z. Altersforsch. 10, 149 (1956). - 11. CAGLI, V., Boll. Soc. ital. Biol. sperim. 30, 273 (1954).
12. Martini, V., Boll. Soc. ital. Biol. sperim. 31, 740 (1955). 13. Berg, G., K. Kimbel und F. Scheiffarti, Naturwissenschaften 42, 51 (1955). - 14. Pilí́ro, S. J. und B. PANSKx, Arch. Biochem. Biophysics 66, 454 (1957). - 15. Gleason, Th. und F. Friedberg, Physiol Zool. 26, 95 (1953). - 16. Schmidt, W., Zbl. Gynäk., Heft 5, 179 (1962). - 17. Purandare, B. N., c. sc., International Congress on Gynecology and Obstetrics, Kongreßband, S. 865 Genf (1954). - 18. Suenderhauf, H. und $C_{H}$. WuNderly, Arch. Gynäk., 185, 513 (1955). - 19. GöretzLEFrner, G., Zbl. Gynäk., 84, 2009 (1962). - 20. GÖRETZLEHnER, G., Zbl. Gynäk., 84, 2009 (1962). - 21. Strunz, K. und A. Hock, Die experimentelle diätische Lebernekrose, Steinkopff-Verlag, Darmstadt (1960). - 22. Brenner, G., W. Korte und A. Puck, Endokrinologie, Leipzig 42, 212 (1962).
Dr. rer. nat. G. Brenner Universitäts-Frauenklinik, Hormon-Labor 53 Bonn-Venusberg 